CERN-TH/99-56

SU-ITP-99/12

\title{
Early Inflation and Cosmology in Theories with Sub-Millimeter Dimensions"̋
}

\author{
Nima Arkani-Hamed ${ }^{a}$, Savas Dimopoulos $^{b}$, Nemanja Kaloper $^{b}$, \\ and John March-Russell ${ }^{c}$ \\ ${ }^{a}$ SLAC, Stanford University, Stanford, CA 94309, USA \\ ${ }^{b}$ Physics Department, Stanford University, Stanford CA 94305, USA \\ ${ }^{c}$ Theory Division, CERN, CH-1211, Geneva 23, Switzerland
}

\begin{abstract}
We discuss early cosmology in theories where the fundamental Planck mass is close to the $\mathrm{TeV}$ scale. In such theories the standard model fields are localized to a $(3+$ 1)-dimensional wall with $n$ new transverse sub-millimeter sized spatial dimensions. The topic touched upon include: early inflation that occurs while the size of the new dimensions are still small, the spectrum and magnitude of density perturbations, the post-inflation era of contraction of our world while the internal dimensions evolve to their final "large" radius, and the production of gravitons in the bulk during these two eras. The radion moduli problem is also discussed.
\end{abstract}

${ }^{*}$ Talk presented by Savas Dimopoulos at COSMO98. The work of SD an NK is supported in part by NSF grant PHY-9870115. The work of JMR is supported in part by an A.P. Sloan Foundation Fellowship. 
Following Refs. [1, 2, 3], there has been much recent interest in the possibility that the fundamental Planck scale $M_{*}$ is close to a TeV 匹, 5, 6, 7, 8, 9, 10]. The weakness of gravity at long distances is then explained by $n$ new "large" dimensions, the (present) size, $b_{0}$, of which is given by the Gauss Law relation $M_{\mathrm{pl}}^{2}=\left(b_{0}\right)^{n} M_{*}^{n+2}$ where $M_{\mathrm{pl}}=2 \times 10^{18} \mathrm{GeV}$. Taking $M_{*} \sim 1 \mathrm{TeV}$ implies the case $n=1$ is excluded since $b_{0} \sim 10^{13} \mathrm{~cm}$, while for $n \geq 2$ however, $b_{0} \lesssim 1 \mathrm{~mm}$, which is the distance where our present experimental knowledge of gravitational strength forces ends. The success of the standard model $(\mathrm{SM})$ up to $\sim 100 \mathrm{GeV}$ implies that the SM fields can not feel these extra dimensions; they must be localized to a $3 \mathrm{~d}$ wall, or "3-brane", in the higher dimensional space. The most attractive possibility for localizing the SM fields to the brane is to employ the D-branes that naturally occur in string theory [11, 2]; this approach has the advantage of being formulated within a consistent quantum theory of gravity. However, the most important question is whether this framework is experimentally invalidated: In Ref. [3], laboratory, astrophysical, and cosmological constraints were found not to exclude these ideas.

Here we will summarize our understanding of inflation and other features of early universe cosmology in such models [12]. In particular we are concerned with three very important questions: 1) How does inflation arise in these models, or is some alternative necessary? 2) How do the new spatial dimensions evolve to their current stabilized value? 3) Does either inflation or the internal evolution produce too much matter in the bulk? Additionally, is there an equivalent of the Polonyi or moduli problem?

\section{Inflation}

The most attractive models of inflation occur while the internal dimensions are still small, far away from their final stabilized value $b_{0}$ [12]. Concretely, the basic story is:

- The quantum creation of the universe takes place with the initial size of all dimensions close to the fundamental Planck scale $M_{*}^{-1}$.

- A prolonged period of inflation in a direction parallel to our brane takes place. The approximately scale-invariant nature of the observed primordial perturbation

\footnotetext{
${ }^{\dagger}$ See also the companion contribution Ref. 13 .

${ }^{\ddagger}$ In particular, we avoid all the problems [8, 9] which are met if inflation occurs after the new dimensions reach their current, large, size. Also note that post-stabilization inflation cannot explain the age of the universe as wall-only inflation cannot begin before $t \sim H^{-1} \sim M_{\mathrm{pl}} / M_{*}^{2}>>M_{*}^{-1}$, when the universe is already very large and old [9, 13].
} 
spectrum implies that, during inflation, the internal dimensions must expand more slowly than the universe on the wall. Thus we are led to consider a form of asymmetric inflationary expansion of the higher-dimensional world $\left(b(t)=b_{I}\right.$ is essentially static). Since the internal dimensions are small the effective 4dimensional Newton's constant is large: $G_{\mathrm{N} \text {,initial }}=1 / b_{I}^{n} M_{*}^{n+2} \simeq 1 / M_{*}^{2}$.

- Thus the Hubble constant during this initial period of inflation can be large even though the energy density is quite small, $\langle V\rangle \leq \mathrm{TeV}^{4}$,

$$
H_{\mathrm{infl}}^{2} \simeq \frac{\langle V\rangle}{6 b_{I}^{n} M_{*}^{(n+2)}} \simeq \frac{\langle V\rangle}{M_{*}^{2}}
$$

Therefore inflation can be rapid, and moreover, the density perturbations can be large, being determined to be

$$
\frac{\delta \rho}{\rho}=\frac{5}{12 \pi \sqrt{2 n(n-1)}} \frac{H_{\mathrm{infl}}}{M_{*}\left(M_{*} b_{I}\right)^{n / 2} S},
$$

where $S$ is a potential-dependent parameter that encapsulates both the duration of $a(t)$ inflation and the deviation of the perturbation spectrum from scaleinvariant Harrison-Zeldovich. (We argue that $S \lesssim 1 / 50$.)

- Specifically, the deviation of the spectral index $n_{\rho}$ of density perturbations $\delta \rho / \rho$ from scale invariance is given by:

$$
n_{\rho}-1 \simeq-\frac{n(n+2)}{2}\left(S^{2}+S T\left(b / b_{I}-1\right)^{2}\right)
$$

where $T$ is another potential-dependent parameter. Thus to have sufficient scale invariance we need just mildly tune $S, T \lesssim 1 / 50$, or alternatively $S \lesssim 10^{-3}$ and $T \sim 1$. Similarly, the number of efolds is given by

$$
N_{e} \simeq \frac{1}{S+T}\left(\frac{2 \sqrt{T} \tan ^{-1}(1 / \sqrt{S})}{\sqrt{S}}-\log (1+1 / S)+2 \log (1+1 / \sqrt{T})\right)
$$

which for $N_{e} \gtrsim 100$ is actually slightly more stringent than (3).

- By going to an effective 4d theory on the wall where $b(t)$ appears as a Brans-Dicke like field (in Einstein frame), it is possible to see that the conditions on $S$ and $T$ are nothing but the usual slow-roll conditions for a scalar-gravity theory.

- In the minimal approach, the inflaton field is just the moduli describing the size of the new dimensions (the radion field of [6]), the role of the inflationary potential 
being played by the stabilizing potential of this internal space. In the case of a wall-localized inflaton, this early inflation might even result from the electroweak phase transition, in which case the inflaton is the Higgs. Actually, an important remark in this regard is that when the internal dimensions are small $b_{I} \sim M_{*}^{-1}$ the distinction between on-the-wall and off-the-wall physics is not meaningful: e.g., the inflationary features in $V(b)$ at small $b$ could be due to Higgs physics on the wall.

\section{Evolution towards stabilization point}

Under quite general conditions, the inflationary era is followed by an epoch where the scale factor of our brane-universe undergoes a slow contraction while the internal dimensions expand towards their final stabilized value. Even with the inclusion of a potential for $b$, it is possible to exactly solve for the evolution during this epoch, which generalizes the usual vacuum Kasner solutions. The history can be summarized as:

- Wall inflation ends, and simultaneously the radion starts to evolve to its' minimum at $b_{0}$. Almost immediately our scale factor $a(t)$ begins to contract. Both $a$ and $b$ have determined (subluminal) power-law dependence on time.

- At generic values of $b$ away from the stabilization point the potential $V(b)$ can be approximated by $V=W b^{-p}$ ( $V$ is the effective 4 d potential, $[W]=4-p$ ). [1] Then the asymptotic form of the exact solutions places an upper bound on the amount of contraction of the brane as a function of $b$ :

$$
\frac{a_{f}}{a_{i}} \leq\left(\frac{b_{i}}{b_{f}}\right)^{\zeta}
$$

where the parameter $\zeta$ is given by $\left(\Delta=6 n-4 n p-n^{2}-p^{2}\right)$

$$
\zeta=\frac{n(n+p-2)}{2(2 n+p)} \quad \text { for } \Delta>0, \quad \zeta=\frac{3 n-\sqrt{3 n(n+2)}}{6} \text { for } \Delta<0 .
$$

- Remarkably this epoch of contraction ends. There are two generic possibilities for how this can happen: The first involves the reheating of the wall. Contraction of $a(t)$ stops and reverses when $\rho_{\text {wall }}$ satisfies

$$
\rho_{\text {wall }}=n(n-1) M_{*}^{n+2} b^{n} H_{b}^{2} \equiv \rho_{b}
$$

\footnotetext{
$\S^{\S}$ This section contains material in addition to that presented at COSMO98.

"An additional logarithmic dependence of $V$ on $b$ is quite possible and harmless.
} 
Here $\rho_{b}$ is the radion (kinetic) energy. Interestingly there is a model-independent form of such reheating that results from the primordial $\rho_{\text {wall }}$ left over from the inflationary epoch. If there is sufficient $a(t)$ contraction, this blue-shifting de Sitter phase remnant $\rho_{\text {wall }}$ can become comparable to $\rho_{b}$ before $b$ reaches the stabilization point, $b_{0}$. It is also possible that a form of reheating takes place on the wall which is totally unconnected with the contraction of $a(t)$, but that again leads to $\rho_{\text {wall }} \geq \rho_{b}$. Possibilities in this class include the decay of some metastable state on the wall, or the collision of some other brane with our brane. After the contraction of $a(t)$ reverses, the radion and wall-localized energy densities scale together until the stabilization point is reached. The second case is where $b(t)$ reaches the stabilization point before $\rho_{\text {wall }}=\rho_{b}$. By going to the Einstein frame it is possible to show that once stabilization of $b$ occurs, the period of $a(t)$ contraction automatically ceases.

- The total amount of contraction of $a(t)$ is bounded and varies between at most 7 efoldings in the case of $n=2$ to at most 12 efoldings when $n=6$.

\section{Bulk graviton production, and moduli problem?}

Naively one would worry that during the era of radion evolution the bulk becomes full of (dangerous) gravitons. There are actually two slightly different issues here: i) The energy density in Kaluza-Klein (KK) excitations of the graviton in the bulk, and, ii) the energy density in the (would-be) zero mode of the bulk graviton, namely the radion. The constraints on the energy density in KK excitations is actually more severe than that on the radion energy density, which just comes from overclosure. The reason for this is the diffuse gamma-ray background constraint arising from KK decays [3]. Again an executive summary of the relevant arguments is:

- A time-dependent gravitational field can produce particles from the vacuum. Including this, the equation for the effective 4d KK energy density becomes

$$
\dot{\rho}_{K K}+3 H_{a} \rho_{K K}+H_{b} \rho_{K K}=H^{5} .
$$

- From this, and the expressions for $a(t)$ and $b(t)$ during the contraction phase, one can show that the dominant contribution to bulk KK graviton production arises from early times. In fact $\rho_{K K \text {,final }}$ differs from the final energy density of the blue-shifted wall-localized radiation at the end of the epoch of contraction only 
in that it is further suppressed by a factor of $\left(a_{f} / a_{i}\right)\left(b_{i} / b_{f}\right)$, which comes from the fact that the KK gravitons are red-shifted by the bulk expansion, and only concentrated and not blue-shifted by the wall contraction. Thus we get

$$
\frac{\rho_{\mathrm{KK}, \mathrm{f}}}{\rho_{\mathrm{wall}, \mathrm{f}}} \leq\left(\frac{a_{f} b_{i}}{a_{i} b_{f}}\right) \lesssim\left(10^{1-30 / n}\right)^{1+\zeta},
$$

using the conservative estimates $b_{i} \sim 10 M_{*}^{-1}$ and $b_{f}=b_{0}$. Evaluating this in, for example, the case of the simple Kasner contraction with exponents given in (6) leads to $\rho_{K K} / \rho_{\text {wall }}$ varying between $3 \times 10^{-17}$ for $n=2$ to $1 \times 10^{-8}$ for $n=6$.

- This shows that the effective temperature of the KK gravitons is well below the diffuse gamma-ray bound, even before any dilution necessary to solve the radion moduli problem. It also demonstrates that the vast majority of the energy in the bulk is in the motion of the zero mode radion $\rho_{b}$, rather than in the bulk KK modes. This is simply because that $\rho_{b} \simeq \rho_{\text {wall }}$ is the natural circumstance at the end of the epoch of contraction.

We now turn to the radion moduli problem:

- Finally around the stabilization point $b_{0}$ the radion field starts to oscillate freely. Since this energy density scales as $1 / a^{3}$, and the wall-to-radion energy densities are initially comparable at the start of the oscillation era, the radion energy eventually dominates the total energy density.

- The most serious question that early universe cosmology presents in the worldas-a-brane scenario is how do we dilute this energy in radion oscillations to an acceptable level. The radion is long-lived, its' decay width back to light wall states being given by

$$
\Gamma_{\varphi} \simeq \frac{m_{\varphi}^{3}}{M_{\mathrm{pl}}^{2}}
$$

We thus require some dilution in the radion energy density, either by a short period of late inflation followed by reheating, or by a delayed reheating after $\rho_{b}$ has sufficiently red-shifted. The amount of dilution of the radion energy density that we require is relatively modest, given roughly by $10^{-7}$, so that only about 5 efolds of late inflation are needed.

"This is increased if there are many branes in the bulk. The "brane crystallization" mechanism of stabilization requires $N_{\text {wall }} \simeq\left(M_{\mathrm{pl}} / M_{*}\right)^{2(n-2) / n}$ branes in the bulk. If each of these have $O(1)$ light modes then the total decay width to all branes is greatly enhanced. 


\section{Conclusions}

We have argued that early inflation when the internal dimensions are still small can successfully accomplish all that is required of inflation, including generation of suitable $\delta \rho / \rho$ without the unpleasant introduction of very light or fine-tuned wall fields. Remarkably the era of post-inflation brane-contraction is harmless, and automatically ends via a "Big Bounce". During the phase of $b(t)$ evolution to the stabilization point, the production of bulk gravitons by the time-varying metric remains completely suppressed, ensuring that the bulk is very cold at, and after, the stabilization of the internal dimensions. The primary remaining issue is the radion moduli problem, which is no more severe than in gauge-mediated supersymmetry breaking models. Overall, then, early universe cosmology in these models is quite interesting!

\section{References}

[1] N. Arkani-Hamed, S. Dimopoulos and G. Dvali, Phys. Lett. B429, 263 (1998).

[2] I. Antoniadis, N. Arkani-Hamed, S. Dimopoulos and G. Dvali, Phys. Lett. B436, 257 (1998).

[3] N. Arkani-Hamed, S. Dimopoulos and G. Dvali, hep-ph/9807344.

[4] I. Antoniadis, Phys. Lett. B246 (1990) 377; I. Antoniadis and K. Benakli, Phys. Lett. B326 (1994) 69; I. Antoniadis, K. Benakli and M. Quiros, Phys. Lett. B331 (1994) 313.

[5] P. Horava and E. Witten, Nucl. Phys. B460 (1996) 506; E. Witten Nucl. Phys. B471 (1996) 135; J.D. Lykken, Phys. Rev. D54 (1996) 3693; E. Caceres, V.S. Kaplunovsky and I.M.Mandelberg, Nucl. Phys. B493 (1997) 73.

[6] N. Arkani-Hamed, S. Dimopoulos and J. March-Russell, hep-th/9809124.

[7] K. Dienes, E. Dudas and T. Gherghetta, Phys. Lett. B436, 55 (1998); Nucl. Phys. B537 (1999) 47; G. Shiu and S.H. Tye, Phys. Rev. D58, 106007 (1998); C. Bachas, hep-ph/9807415; R. Sundrum, hep-ph/9805471 and hep-ph/9807348; P. Argyres, S. Dimopoulos and J. March-Russell, hep-th/9808138; Z. Kakushadze and S.-H. Tye, hep-th/9809147; K. Dienes, et al., hep-ph/9809406; K. Benakli, hep-ph/9809582; L. Randall and R. Sundrum, hep-th/9810155; G.F. Giudice, 
R. Rattazzi and J.D. Wells, hep-ph/9811291; S. Nussinov and R. Shrock, hepph/9811323; E.A. Mirabelli, M. Perelstein and M.E. Peskin, hep-ph/9811337; T. Han, J.D. Lykken and R. Zhang, hep-ph/9811350; N. Arkani-Hamed and S. Dimopoulos, hep-ph/9811353; J.L. Hewett, hep-ph/9811356; Z. Berezhiani and G. Dvali, hep-ph/9811378; K.R. Dienes, E. Dudas and T. Gherghetta, hepph/9811428; N. Arkani-Hamed, et al., hep-ph/9811448; I. Antoniadis and C. Bachas, hep-th/9812093; Z. Kakushadze, hep-th/9812163.

[8] K. Benakli and S. Davidson, hep-ph/9810280; D. Lyth, hep-ph/9810320.

[9] N. Kaloper and A. Linde, hep-th/9811141.

[10] G. Dvali and S.H. Tye, hep-ph/9812483.

[11] See for example: J. Polchinski, TASI lectures on D-branes, hep-th/9611050; C. Bachas, Lectures on D-branes, hep-th/9806199.

[12] N. Arkani-Hamed, S. Dimopoulos, N. Kaloper, and J. March-Russell, hepph/9803224.

[13] N. Kaloper, talk at COSMO98, and contribution to this volume. 